\title{
Abundance and patterns of rarity of Polylepis birds in the Cordillera Vilcanota, southern Perú: implications for habitat management strategies
}

\author{
HUW LLOYD
}

\section{Summary}

Point count distance sampling surveys were conducted at three sites in the Cordillera Vilcanota to determine whether variation in high-Andean species richness, diversity and abundance was a reflection of Polylepis habitat quantity. Bird community and abundance measures revealed that there was considerably variation in bird species richness, diversity and mean encounter rates between large, medium, and small forest patches. Densities of Polylepis-dependent bird species (including five globally-threatened and eight restricted-range species) were greater in larger forest patches and differed significantly between different patch size categories. Density estimates for matrix-dependent species were higher in smaller Polylepis patches indicating that the matrix exerts an influence on bird species composition and abundance in remnant Polylepis forests, particularly smaller patches. Comparison of lowland forest habitat specialists using three categories of rarity revealed that between $19-22 \%$ of all Polylepis-dependent species were intrinsically rare within larger forest patches, and a greater number $(34-74 \%)$ were rare in smaller forest patches. Population estimates for all species, in particular for all threatened species, were extremely low, numbering $\leqslant 10$ individuals at nine of the ten sites examined. The results suggest that declines in the densities of certain Polylepis birds may be predictable following habitat loss and that these patterns of rarity should govern population recovery goals through appropriate habitat restoration strategies. Such strategies are urgently required and must be designed to prevent further habitat loss, and to increase Polylepis habitat quantity to boost threatened bird populations in the Cordillera Vilcanota.

\section{Resumen}

Evaluaciones de distancias de puntos de conteo fueron conducidas en tres lugares en la Cordillera Vilcanota para determinar si la variación en la riqueza, diversidad y abundancia de las especies de los Andes altos, era un reflejo de la cantidad de hábitats de Polylepis. La comunidad de aves y su abundancia revelaron que hubo una considerable variación en la riqueza de especies, diversidad y la media encontrada en rangos parches de bosque comprendidos entre grandes, medios y pequeños. La densidad de especies de aves dependientes de Polylepis (incluyendo cinco amenazados globalmente y ocho especies con rango restringido) fueron mayores en parches grandes de bosque y difirieron significativamente entre diferentes categorías de tamaños de parche. La densidad estimada para especies matriz-dependientes fue mayor en pequeños parches de Polylepis indicando que la matriz ejerce una influencia en la composición de especies de aves y abundancia en vestigios de bosques de Polylepis, particularmente parches pequeños. En comparación con especialistas de hábitat de bosque bajo, empleando tres categorías de rareza revelaron que entre $19-22 \%$ de todos las especies dependientes de Polylepis fueron intrínsicamente raras dentro de parches grandes de bosque, y un número mayor $(34-74 \%)$ 
fueron raras en los parches pequeños de bosque. La población estimada para todas las especies, en particular para todas las especies amenazadas, fue extremadamente baja, contando $\leqslant$ 10 individuos en nueve de los 10 sitios examinados. El resultado sugiere que la disminución en la densidad de ciertas aves de Polylepis se podría predecir después de la pérdida de hábitat y que esos patrones de rareza podrían servir como objetivos para la recuperación de la población a través de estrategias de restauración de hábitats apropiados. Este tipo de estrategias son requeridas con urgencia y deberían estar diseñadas para prevenir futuras pérdidas de hábitats, incrementar la cantidad de hábitat de Polylepis para aumentar la población de aves amenazadas en la Cordillera Vilcanota.

\section{Introduction}

The number and type of bird species that inhabit isolated forest patches is largely dependent on the area of the patch (Ambuel and Temple 1983, Blake and Karr 1987). Forest patches are often surrounded by an 'inhospitable' matrix of disturbed habitat e.g. agricultural land, with significantly different habitat structure, floral and faunal composition (e.g. Stouffer and Bierregaard 1995, Gascon et al. 1999, Driscoll 2005). This concept is central to many conservation strategies (Diamond 1975, Fischer et al. 2005) because forest-dependent species are often restricted to only the largest remnant forest patches (Blake and Karr 1984, 1987). This is often a result of the smaller forest patches being dominated by ecological generalists that are able to forage in the surrounding matrix habitat (Ambuel and Temple 1983, Laurance 1994, Antongiovanni and Metzger 2005).

Many threatened Neotropical forest-dependent bird species are habitat specialists with narrow geographical distributions (BirdLife International 2004). Conservation of these forest habitat specialists may best be approached by identifying and conserving key areas of forest habitat, in conjunction with the strategy of conserving the largest forest areas (Kratter 1995). High-Andean Polylepis forests are one of the most threatened forest habitats in Latin America (Fjeldså and Kessler 1996, Stotz et al. 1996, Kessler 2002). As a consequence of ongoing habitat loss and degradation (e.g. Lægaard 1992, Renison et al. 2004, Teich et al. 2005) many of the constituent habitat specialist bird species are restricted to remnant forest patches surrounded by a matrix of either disturbed puna (non-forest) habitat above 3,800 m elevation, or evergreen shrubs and other woody plant species (depending on the level of burning) below this elevation. Furthermore, many of these bird species are presumed to have extremely small populations in the tiny forest patches that remain (Fjeldså 1988, 1993, 2002). In the Cordillera Vilcanota, southern Perú, six globally-threatened high-Andean bird species, and seven other restrictedrange species, are dependent to a degree, on Polylepis woodland and intolerant of the surrounding puna matrix habitat (Lloyd and Marsden in press). For these threatened species intolerant of the matrix habitat, the maintenance of viable populations inside remaining forest patches is the only realistic conservation option. An important consideration is therefore whether these bird species occur in reasonable numbers in forest patches of different sizes.

Density is one of the most important operational terms in ecology (Peters 1991) and is often used to express both the distribution and abundance of species within an ecological community or landscape, and for habitat management recommendations (Smallwood 2001). Density estimates can provide a strong foundation for the conservation of Polylepis forest bird species in Peru, particularly as the current IUCN criteria for ranking the degree of threat to any bird species are highly quantitative (BirdLife International 2004, Lloyd 2004; 2007). Reliable density estimates could allow conservation biologists to make a number of predictions regarding the numbers of birds in unsurveyed highly fragmented areas (Buckland et al. 1993, Jones et al. 1995, Marsden 1999). Furthermore, determining whether or not Polylepis birds are intrinsically lowdensity species in different patch size categories links habitat restoration efforts in areas where Polylepis habitat once existed, to meaningful quantitative ecological units (numbers of 
individuals per unit area of habitat) that are critical for setting realistic population recovery goals and maintaining viable populations (e.g. Tear et al. 1995, Rivera-Milan et al. 2003).

The objective of this paper is to examine Polylepis bird species richness, diversity, and densities in different forest patch size categories and ask whether variation in these factors is a reflection of habitat quantity. Are Polylepis bird species intrinsically rare species? Do patterns of rarity within the Polylepis bird community have significant implications for high Andean habitat management strategies?

\section{Methods}

\section{Study sites}

Polylepis bird communities were surveyed in three valleys in the Cordillera Vilcanota mountain range, Dept. of Cusco, southern Perú (Fig I). Mantanay ( $13^{\circ} 12^{\prime} \mathrm{S}, 72^{\circ} \mathrm{Og}{ }^{\prime} \mathrm{W}$ ) is one of the largest areas of Polylepis racemosa woodland in the Cordillera Vilcanota, located at c. 3,400-4,500 $\mathrm{m}$ elevation, above the village of Yanahuara. The site was surveyed during 67 field days in July 2003, October 2004, and September 2005. Yanacocha $\left(13^{\circ} 17^{\prime} \mathrm{S}, 72^{\circ} \mathrm{O} 2^{\prime} \mathrm{W}\right)$ is another area of P. racemosa woodland, located at $c .3,700-4,500 \mathrm{~m}$ elevation, above the village of Huaocari, and was surveyed for 28 days in October 2003 and June 2004. Laguna Queuñacocha $\left(13^{\circ} 12^{\prime} \mathrm{S}\right.$, $72^{\circ} 10^{\prime} \mathrm{W}$ ) is a small area of P. pepei woodland, located at $c .4,200-4,500 \mathrm{~m}$ elevation above the village of Huilloc (hereafter referred to as Huilloc), and was surveyed during 22 field days in December 2003 and July 2004.

\section{Habitat measurements}

Polylepis forest patches were assigned to one of three size categories: small (o.1 - o.8 ha), medium (4.5-9.0 ha), and large (12.0-31.5 ha) patches. I consider whole patches as potentially containing both forest-interior and forest-edge habitat because of the size and shape of the forest patches. Deforestation throughout the Cordillera Vilcanota has not followed a regular pattern mainly because of the topographical complexity of the region, with only a few high Andean areas still having patches of mature Polylepis forest (closed canopy, large trees etc). Some areas

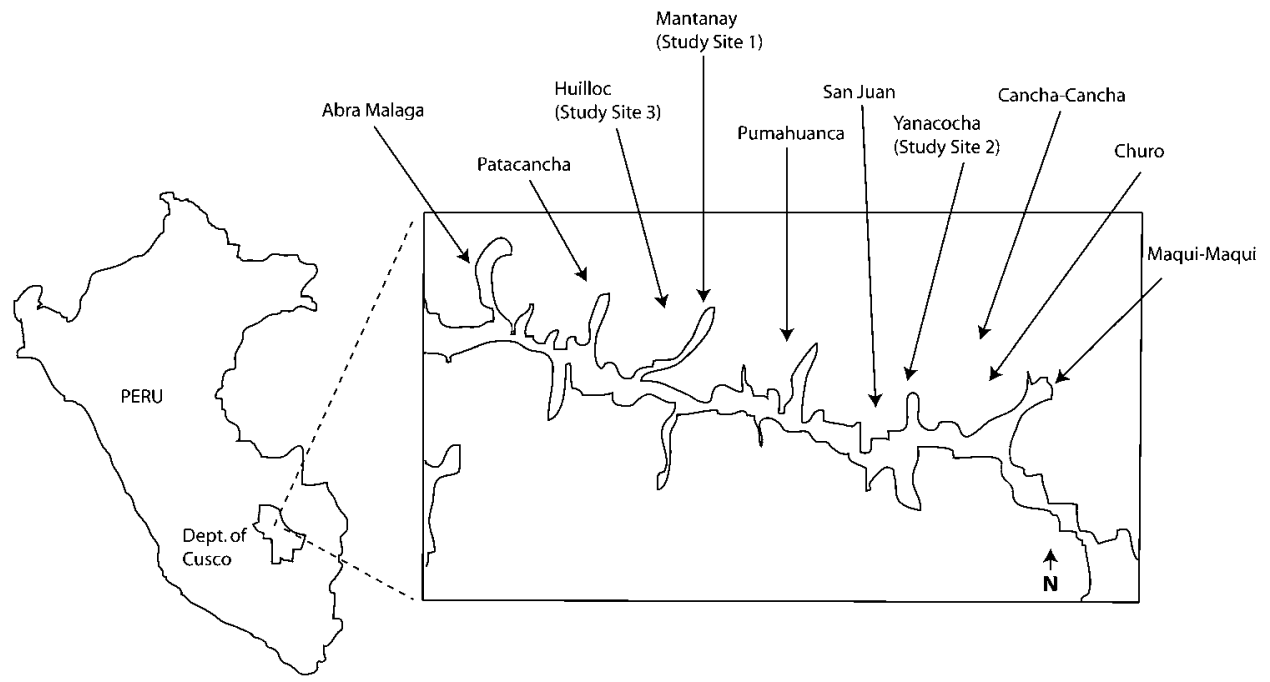

Figure 1. Map showing location of ten areas of Polylepis woodland (including three study sites) situated in the Urubamba valley, Cordillera Vilcanota. 
in the Cordillera Vilcanota have been cut more extensively than others and the amount of regeneration may be very variable. Consequently, most of the forest patches are irregular in shape and habitat quality. Data on estimated forest cover for ten sites in the Cordillera Vilcanota (Fig 1), including the three study sites, refer to land surface cover in Aucca et al. (2001) and are shown in Table 4.

\section{Bird surveys}

Birds were surveyed at plots within Polylepis using a variable circular plot method (Reynolds et al. 1980) adapted by Jones et al. (1995). This distance sampling method allows for differences in detectability between different species, and within a species between different habitats (Buckland et al. 1993, 2001). The method also allows for some unidentified bird contacts, so long as all bird contacts encountered at, and very close to, the recorder are identified (Buckland et al. 1993, 2001). Bird surveys were conducted between o5h3o and 16h30 and only during hours of suitable weather (i.e. in the absence of snow, rain or strong wind). Between June-September in the Cordillera Vilcanota, bird vocal activity begins around $06 \mathrm{~h}_{30}$ and around $0_{5} \mathrm{~h}_{30}$ between October and December and there is no conspicuous peak in high-Andean bird vocal activity at either the pre-dawn or dawn period (pers. obs.) as observed in the Amazonian lowlands (e.g. Terborgh et al. 1990, Lloyd 2004).

I was the only recorder for all bird surveys; with over two years experience of surveying birds using similar methods in Perú, and over four years of experience in high Andean landscapes as a naturalist and bird tour leader, I was familiar with the vocalizations of all of the region's bird species. Following arrival at each plot, I sat quietly for a five minute 'settling down' period (Bibby et al. 200o) before spending twenty minutes recording all bird contacts. Each contact was assigned to one of three categories; seen; heard; or seen and heard, and species and the number of individuals in each group recorded. I then estimated horizontal distance from the centre of the census plot to each contact. Two repeats of each transect were made at each site making a total of 266 point counts. The direction of the surveys along transects was rotated to counter the bias of bird activity and the time of day.

\section{Data analysis}

\section{Bird species richness and diversity}

Bird species richness and diversity were calculated using rarefaction analysis (Simberloff 1972). Rarefaction estimates the number of species from a given sample of point transects based on multiple random sampling (James and Rathburn 1981) and was implemented using EstimateS v.7.5 (Colwell 2005). Sample order was randomised 50 times for each dataset (Lee 2005). Sample species richness was estimated from the sample-based rarefaction curves (Mau Tau; $\mathrm{S}_{\mathrm{obs}}$ ). The bootstrap estimator ( $S_{\text {boot }}$ : Smith and van Belle 1984, Colwell and Coddington 1994) a widely used and easily understood robust estimator (Borgella and Gavin 2005, Lee 2005) was used as a measure of estimated species richness. Bird species diversity was measured using the ShannonWiener index $\left(H^{\prime}\right)$, which takes into account both species richness and the relative abundance of each species (Magurran 2004, Lee 2005).

\section{Density estimates}

Distance data were analyzed using the program DISTANCE v. 4.I (Thomas et al. 2004). Only bird species with $\geqslant 20$ records were considered for the calculation of density estimates, with the majority of species fulfilling the minimum of 40 records required to estimate densities (Buckland et al. 1993). Four key functions (uniform, half-normal hazard rate and negative exponential all with cosine series adjustment) were considered for each analysis. Key function selection was 
evaluated using Akaike's Information Criteria (AIC) and a chi-square statistic was used to assess the 'goodness of fit' of each function (Buckland et al. 1993). Calculation of \% CV was done empirically (e.g. Lloyd 2000, 2004). Repeated point transects increased species sample sizes, providing a more precise estimate of variance and increasing the reliability of the detection function (Buckland et al. 2001, Lee 2005). Distance data were pooled across all sites and patch size categories to produce a single species-specific detection function from which species-specific density estimates were calculated in each forest patch size category (Marsden 1999, Marsden et al. 2001). Pooling records generated a more precise modelling of detectability because the combined AIC values of the separate patch size detection functions were greater than the AIC value for the pooled detection function (Buckland et al. 2001). The shape criteria were examined for heaping or cluster bias and any outliers were right-hand truncated where necessary (Buckland et al. 1993). Determination of actual values for truncation and subsequent grouping of records into distance bands followed visual inspection of detection histograms and checking of \% SE for density estimates under different analysis conditions (Buckland et al. 2001). Encounter rates (number of individuals per point) as an estimate of relative abundance, were calculated for species that did not fulfil the minimum sample size criterion (Lee 2005).

\section{Population estimates}

Forest area data from all the locations were used together with the combined species density estimates to calculate total population estimates, where possible, for each species, using the formula: total population estimate $=$ density estimate $\mathrm{x}$ area (Marsden et al. 2005). Minimum and maximum population sizes were based on the lower- and upper confidence intervals obtained from the density estimates (Jacobs and Walker 1999).

\section{Results}

\section{Bird species richness and diversity}

Both measures of species richness $\left(\mathrm{S}_{\mathrm{obs}}\right)$ and the Shannon-Wiener index $\left(H^{\prime}\right)$ were fairly similar across all three forest patch size categories (Table 2). Identical numbers of globally-threatened and restricted-range species were recorded in large and medium patches at Mantanay and Yanacocha, and large patches at Huilloc but fewer species in both categories were recorded in medium and small patches at Huilloc. Species richness $\left(\mathrm{S}_{\text {obs }}\right.$ and $\left.\mathrm{S}_{\text {boot }}\right)$ was greater in large and small forest patches at Mantanay and greater in medium forest patches at Yanacocha. The Shannon-Wiener index of species diversity $\left(H^{\prime}\right)$ was greater in large patches at Mantanay and Huilloc, and in medium forest patches at Yanacocha. Fewer threatened and restricted-range species were recorded in medium and small patches at Huilloc.

\section{Encounter rates and density estimates}

Generally, the mean encounter rate was higher in large patches, than in medium or small patches (Table 2). Mean encounter rate of birds in large and small forest patches across all three sites was highest at Huilloc, and higher in medium forest patches at Yanacocha.

When sample sizes were pooled across all three sites and all three forest patch size categories, I was able to calculate total Polylepis density estimate for all 36 species (Table 3). Most species (22) had density estimates < 10 individuals $\mathrm{km}^{-2}$; eight species had density estimates ranging between $10-20$ individuals $\mathrm{km}^{-2}$; five species had density estimates $>20$ individuals $\mathrm{km}^{-2}$; and only one species had a density estimate $>30$ individuals $\mathrm{km}^{-2}$. The most abundant species was A. castelnaudii, and the rarest was $O$. oenanthoides. The most abundant globally threatened species was $L$. xenothorax, and the rarest $C$. aricomae.

There was a significant difference in the density of all bird species between each patch size category (mean density estimate large patch $=7.0 \pm 6.6$; medium $=5.2 \pm 6.2$; small $=4.5 \pm$ 
9.2 Kruskal-Wallis test; $\chi_{2}^{2}=10.8, P<0.01$ ). A. castelnaudii (restricted-range) was the most abundant species in both large and medium forest patches and $G$. andicola (restricted-range) was the rarest in both large and small patches. In small forest patches the most abundant species was P. unicolor (matrix-dependent) and the rarest in medium patches was C. aricomae (Polylepisdependent and globally-threatened). Generally, threatened and restricted-range species were more abundant in large forest patches than in medium or small forest patches, except for $A$. castelnaudii and G. andicola which were more abundant in medium patches.

\section{Estimates of population sizes}

Population estimates for all species at the ten locations are shown in Table 4. Estimates were higher in areas with greater forest cover (e.g. Mantanay) and smaller in areas with the least

Table 1. Bird species recorded during point count distance sampling surveys at three Polylepis sites in the Cordillera Vilcanota. Conservation status * follows BirdLife International (2004). Patterns of habitat occupancy $\dagger$ from Lloyd (2007) and refer to the three study sites.

\begin{tabular}{|c|c|c|}
\hline Species & Status * & Habitat Occupancy $\uparrow$ \\
\hline Aglaeactis castelnaudii & Restricted-range & Polylepis forest \\
\hline Oreotrochilus estella & Not threatened & Polylepis-matrix interface \\
\hline Chalcostigma stanleyi & Not threatened & Polylepis forest \\
\hline Colaptes rupicola & Not threatened & Matrix habitat \\
\hline Cinclodes aricomae & Critical & Polylepis forest \\
\hline Cinclodes fuscus & Not threatened & Polylepis-matrix interface \\
\hline Leptasthenura yanacensis & Near-threatened & Polylepis forest \\
\hline Leptasthenura xenothorax & Endangered & Polylepis forest \\
\hline Cranioleuca albicapilla & Restricted-range & Polylepis forest \\
\hline Asthenes ottonis & Restricted-range & Polylepis forest \\
\hline Asthenes humilis & Not threatened & Matrix habitat \\
\hline Asthenes urubambensis & Not threatened & Polylepis forest \\
\hline Asthenes virgata & Restricted-range & Polylepis-matrix interface \\
\hline Grallaria andicola & Restricted-range & Polylepis forest \\
\hline Scytalopus simonsi & Restricted-range & Polylepis forest \\
\hline Mecocerculus leucophrys & Not threatened & Polylepis forest \\
\hline Anairetes alpinus & Endangered & Polylepis forest \\
\hline Anairetes parulus & Not threatened & Polylepis forest \\
\hline Ochthoeca rufipectoralis & Not threatened & Polylepis forest \\
\hline Ochthoeca oenanthoides & Not threatened & Polylepis-matrix interface \\
\hline Troglodytes aedon & Not threatened & Polylepis forest \\
\hline Turdus chiguanco & Not threatened & Polylepis forest \\
\hline Turdus fuscater & Not threatened & Polylepis forest \\
\hline Conirostrum cinereum & Not threatened & Polylepis forests \\
\hline Conirostrum ferrugineiventre & Restricted-range & Polylepis forests \\
\hline Oreomanes fraseri & Near-threatened & Polylepis forests \\
\hline Xenodacnis parina & Restricted-range & Polylepis forests \\
\hline Diglossa brunneiventris & Not threatened & Polylepis forests \\
\hline Zonotrichia capensis & Not threatened & Matrix habitat \\
\hline Phrygilus punensis & Not threatened & Polylepis-matrix interface \\
\hline Phrygilus unicolor & Not threatened & Matrix habitat \\
\hline Phrygilus plebejus & Not threatened & Matrix habitat \\
\hline Idiopsar brachyurus & Not threatened & Polylepis-matrix interface \\
\hline Catamenia inornata & Not threatened & Polylepis-matrix interface \\
\hline Carduelis atrata & Not threatened & Polylepis-matrix interface \\
\hline Carduelis crassirostris & Restricted-range & Polylepis forest \\
\hline
\end{tabular}


Table 2. Bird species richness and diversity of different Polylepis forest patch categories in the three valleys in the Cordillera Vilcanota, southern Peru. $\mathrm{S}_{\mathrm{obs}}=$ sample species richness from sample-based rarefaction curves. $\mathrm{S}_{\text {boot }}=$ bootstrap estimator. $\mathrm{H}^{\prime}=$ Shannon-Wiener index of bird diversity (see Data analysis).

\begin{tabular}{|c|c|c|c|c|c|c|c|c|c|}
\hline & \multicolumn{3}{|l|}{ LARGE } & \multicolumn{3}{|l|}{ MEDIUM } & \multicolumn{3}{|l|}{ SMALL } \\
\hline & Mantanay & Yanacocha & Huilloc & Mantanay & Yanacocha & Huilloc & Mantanay & Yanacocha & Huilloc \\
\hline Number of point counts & 44 & 28 & 28 & 30 & 20 & 6 & 40 & 24 & 26 \\
\hline Mean encounter rate & 1.67 & 1.58 & 2.03 & 1.11 & 1.15 & 1.08 & 1.79 & 1.37 & 2.15 \\
\hline Species richness $\left(\mathrm{S}_{\mathrm{obs}}\right)$ & $35 \pm 1.66$ & $26 \pm 2.07$ & $27 \pm 0.67$ & $27 \pm 1.32$ & $29 \pm 2.62$ & $19 \pm 3.37$ & $29 \pm 1.83$ & $21 \pm 0.89$ & $23 \pm 2.13$ \\
\hline Species richness $\left(\mathrm{S}_{\text {boot }}\right)$ & $37 \pm 0.61$ & $27 \pm 0.57$ & $28 \pm 0.48$ & $30 \pm 0.97$ & $33 \pm 1.21$ & $24 \pm 4.36$ & $33 \pm 0.99$ & $23 \pm 0.49$ & $26 \pm 0.99$ \\
\hline Species diversity $\left(H^{\prime}\right)$ & $2.98 \pm 0.01$ & $2.76 \pm 0.01$ & $2.90 \pm 0.03$ & $2.84 \pm 0.04$ & $2.93 \pm 0.03$ & $2.46 \pm 0.06$ & $2.63 \pm 0.03$ & $2.77 \pm 0.03$ & $2.79 \pm 0.04$ \\
\hline $\begin{array}{l}\text { Number of threatened } \\
\text { species (restricted-range) }\end{array}$ & $6(9)$ & $6(8)$ & $6(6)$ & $6(9)$ & $6(9)$ & $4(5)$ & $6(9)$ & $6(9)$ & $3(5)$ \\
\hline
\end{tabular}


Table 3. Encounter rates and density estimates $\pm \% \mathrm{CV}$, with $95 \%$ confidence intervals for 36 commonly encountered bird species in different forest patch size categories, at the three sites. Density estimates (DE) are expressed as number of individuals $\mathrm{km}^{-2}$ Polylepis forest. Encounter rates ${ }^{*}$ expressed as total number of individuals recorded per point count $( \pm S D)$.

\begin{tabular}{|c|c|c|c|c|}
\hline Species & $\begin{array}{l}\text { Total Polylepis DE } \pm \% \mathrm{CV} \\
(95 \% \mathrm{CI})\end{array}$ & $\begin{array}{l}\text { Large patch } \mathrm{DE} \pm \% \mathrm{CV} \\
(95 \% \mathrm{CI})\end{array}$ & $\begin{array}{l}\text { Medium patch DE } \pm \% \mathrm{CV} \\
(95 \% \mathrm{CI})\end{array}$ & $\begin{array}{l}\text { Small patch DE } \pm \% \mathrm{CV} \\
(95 \% \mathrm{CI})\end{array}$ \\
\hline Aglaeactis castelnaudii & $47.8 \pm 17.9(33.6-68.0)$ & $23.6 \pm 28.0(12.4-45.0)$ & $25.6 \pm 30.3(13.3-49.3)$ & $0.20 \pm 0.40 *$ \\
\hline Oreotrochilus estella & $10.8 \pm 39.6(5.0-23.5)$ & $0.09 \pm 0.29^{*}$ & $0.04 \pm 0.19^{*}$ & $0.16 \pm 0.36^{*}$ \\
\hline Chalcostigma stanleyi & $12.3 \pm 36.5(6.0-25.1)$ & $6.4 \pm 60.5(2.0-21.1)$ & $5.7 \pm 45.6(2.1-15.4)$ & $0.08 \pm 0.27^{*}$ \\
\hline Colaptes rupicola & $8.2 \pm 156.8(0.8-84.6)$ & $0.04 \pm 0.20^{*}$ & $0.07 \pm 0.26^{*}$ & $0.19 \pm 0.39 *$ \\
\hline Cinclodes aricomae & $1.0 \pm 43.0(0.4-2.1)$ & $2.5 \pm 73.7(0.6-10.8)$ & $0.5 \pm 40.5(0.2-1.1)$ & $0.01 \pm 0.11 *$ \\
\hline Cinclodes fuscus & $6.3 \pm 43.0(2.8-14.2)$ & $2.1 \pm 29.5(1.0-4.1)$ & $0.6 \pm 43.1(0.2-1.9)$ & $10.9 \pm 51.3(4.1-28.8)$ \\
\hline Leptasthenura yanacensis & $11.9 \pm 28.6(6.8-20.8)$ & $11.6 \pm 43.3(4.6-29.4)$ & $8.9 \pm 45.5(3.3-24.3)$ & $5.1 \pm 21.4(3.0-8.5)$ \\
\hline Leptasthenura xenothorax & $15.9 \pm 23.0(10.2-24.9)$ & $25.3 \pm 15.1(18.8-34.1)$ & $9.6 \pm 21.7(5.9-15.7)$ & $0.07 \pm 0.25^{*}$ \\
\hline Cranioleuca albicapilla & $13.9 \pm 28.4(8.0-24.2)$ & $20.1 \pm 55.0(7.1-57.2)$ & $13.1 \pm 36.6(6.4-27.1)$ & $0.08 \pm 0.27^{*}$ \\
\hline Asthenes ottonis & $8.5 \pm 62.5(2.4-29.8)$ & $16.3 \pm 39.0(7.7-34.4)$ & $5.2 \pm 51.2(1.5-17.9)$ & o \\
\hline Asthenes humilis & $1.7 \pm 31.3(1.3-2.4)$ & o & $0.04 \pm 0.19 *$ & $2.4 \pm 36.8(1.1-4.9)$ \\
\hline Asthenes urubambensis & $1.4 \pm 59.3(0.4-4.3)$ & $0.09 \pm 0.29^{*}$ & $0.07 \pm 0.35^{*}$ & $0.08 \pm 0.27^{*}$ \\
\hline Asthenes virgata & $3.4 \pm 42.0(1.5-7.7)$ & $0.11 \pm 0.31 *$ & $0.07 \pm 0.26 *$ & $0.07 \pm 0.25^{*}$ \\
\hline Grallaria andicola & $2.0 \pm 40.8(0.9-4.4)$ & $1.3 \pm 25.8(0.5-3.9)$ & $3.3 \pm 70.7(0.8-12.8)$ & $1.2 \pm 70.4(0.3-4.8)$ \\
\hline Scytalopus simonsi & $3.9 \pm 35.4(2.0-7.9)$ & $6.1 \pm 30.2(3.2-11.4)$ & $2.2 \pm 40.6(0.9-5.1)$ & $0.07 \pm 0.25 *$ \\
\hline Mecocerculus leucophrys & $8.6 \pm 41.3(3.8-19.3)$ & $11.8 \pm 43.8(4.9-28.3)$ & $1.0 \pm 57.7(0.2-5.5)$ & o \\
\hline Anairetes alpinus & $4.9 \pm 31.7(2.6-9.2)$ & $4.3 \pm 41.8(1.8-10.1)$ & $2.6 \pm 48.2(0.9-7.4)$ & $0.04 \pm 0.21 *$ \\
\hline Anairetes parulus & $14.8 \pm 24.2(9.2-24.0)$ & $13.8 \pm 31.6(7.1-26.6)$ & $6.6 \pm 64.1(1.1-41.3)$ & $0.06 \pm 0.23 *$ \\
\hline Ochthoeca rufipectoralis & $11.1 \pm 21.9(7.2-17.1)$ & $5.1 \pm 21.0(3.3-8.0)$ & $5.3 \pm 36.2(2.5-11.2)$ & $0.07 \pm 0.25 *$ \\
\hline Ochthoeca oenanthoides & $0.5 \pm 38.0(0.2-1.0)$ & $0.14 \pm 0.35^{*}$ & $0.23 \pm 0.43^{*}$ & $0.08 \pm 0.27^{*}$ \\
\hline Troglodytes aedon & $5.3 \pm 19.0(3.6-7.7)$ & $8.1 \pm 22.4(5.2-12.9)$ & $3.0 \pm 19.8(1.9-4.5)$ & $2.4 \pm 24.6(1.5-3.9)$ \\
\hline Turdus chiguanco & $1.0 \pm 47.1(0.4-2.2)$ & $0.5 \pm 73.4(0.1-1.9)$ & $2.3 \pm 53.1(0.7-7.8)$ & $0.01 \pm 0.11 *$ \\
\hline Turdus fuscater & $1.9 \pm 48.6(0.7-5.1)$ & $3.7 \pm 34.4(1.9-7.2)$ & $1.5 \pm 31.2(0.8-2.8)$ & $0.01 \pm 0.11 *$ \\
\hline Conirostrum cinereum & $28.3 \pm 38.8(14.8-54.2)$ & $15.1 \pm 45.6(5.1-45.1)$ & $8.4 \pm 30.8(4.5-15.6)$ & $13.4 \pm 50.5(4.7-38.0)$ \\
\hline Conirostrum ferrugineiventre & $14.1 \pm 30.5(7.5-26.4)$ & $14.9 \pm 32.5(7.5-29.4)$ & $8.5 \pm 27.0(4.8-15.1)$ & o \\
\hline Oreomanes fraseri & $16.6 \pm 13.6(12.2-22.5)$ & $22.1 \pm 14.8(16.5-29.6)$ & $12.9 \pm 30.0(6.7-24.9)$ & $5.5 \pm 22.5(3.5-8.6)$ \\
\hline Xenodacnis parina & $17.9 \pm 41.1(7.2-44.5)$ & $23.5 \pm 24.6(14.4-38.6)$ & $10.5 \pm 52.6(2.4-46.5)$ & $0.19 \pm 0.39 *$ \\
\hline Diglossa brunneiventris & $3.3 \pm 36.8(1.6-6.8)$ & $3.9 \pm 61.1(1.0-15.2)$ & $1.0 \pm 48.4(0.1-4.6)$ & $0.04 \pm 0.21 *$ \\
\hline Zonotrichia capensis & $9.6 \pm 31.1(5.2-17.7)$ & $4.3 \pm 49.9(1.5-11.9)$ & $19.9 \pm 37.8(9.1-43.7)$ & $6.3 \pm 34.6(3.1-12.8)$ \\
\hline
\end{tabular}

Asthenes urubambensis

Asthenes virgata

Scytalopus simons

Mecocerculus leucophrys

Ochthoeca rufipectorali

$47.8 \pm 17.9(33.6-68.0)$

.

$1.7 \pm 31.3(1.3-2.4)$

$59.3(0.4-4.3)$

$8.6 \pm 41.3(3.8-19.3)$

$11.1 \pm 21.9(7.2-17.1)$

$28.3 \pm 38.8(14.8-54.2)$

$30.5(7.5-26.4)$

$17.9 \pm 41.1(7.2-44.5)$

$9.6 \pm 31.1(5.2-17.7)$

$4.3 \pm 49.9$ (1.5-11.9)

$19.9 \pm 37.8(9.1-43.7)$ 
Table 3. Continued.

\begin{tabular}{|c|c|c|c|c|}
\hline Species & $\begin{array}{l}\text { Total Polylepis DE } \pm \% \text { CV } \\
(95 \% \text { CI })\end{array}$ & $\begin{array}{l}\text { Large patch DE } \pm \% \mathrm{CV} \\
(95 \% \mathrm{CI})\end{array}$ & $\begin{array}{l}\text { Medium patch DE } \pm \% \mathrm{CV} \\
(95 \% \mathrm{CI})\end{array}$ & $\begin{array}{l}\text { Small patch DE } \pm \% \text { CV } \\
(95 \% \mathrm{CI})\end{array}$ \\
\hline Phrygilus punensis & $5.9 \pm 19.4(4.0-8.7)$ & $5.3 \pm 27.0(2.9-9.5)$ & $15.3 \pm 65.6(3.0-79.2)$ & $11.3 \pm 45.7(4.4-28.9)$ \\
\hline Phrygilus unicolor & $21.3 \pm 33.4(11.1-41.2)$ & $5.4 \pm 52.2(1.9-15.7)$ & $0.02 \pm 0.13^{*}$ & $41.3 \pm 25.2(25.1-68.1)$ \\
\hline Phrygilus plebejus & $8.7 \pm 50.0(3.1-24.3)$ & $6.8 \pm 91.0(0.4-122.0)$ & o & $16.8 \pm 46.6(6.1-45.9)$ \\
\hline Idiopsar brachyurus & $4.5 \pm 75.6(1.0-19.1)$ & $0.01 \pm 0.10^{*}$ & $0.07 \pm 0.26 *$ & $0.19 \pm 0.39$ * \\
\hline Catamenia inornata & $4.3 \pm 21.3(2.8-6.5)$ & $3.9 \pm 25.1(2.3-6.5)$ & $2.2 \pm 58.8(0.0-239.2)$ & $13.7 \pm 65.3(3.5-52.6)$ \\
\hline Carduelis atrata & $8.8 \pm 24.3(5.4-14.6)$ & $5.4 \pm 46.8(1.8-16.4)$ & $1.5 \pm 42.2(0.5-4.1)$ & $31.4 \pm 45.8(12.6-78.3)$ \\
\hline Carduelis crassirostris & $9.9 \pm 22.9(6.2-15.7)$ & $11.4 \pm 39.7(5.0-25.8)$ & $9.1 \pm 33.3(4.4-18.7)$ & $0.04 \pm 0.21 *$ \\
\hline
\end{tabular}


Table 4. Population estimates of 36 species of Polylepis birds in ten locations in the Cordillera Vilcanota. Estimates of forest cover from Aucca et al. (2001). Figures in parentheses represent minimum and maximum population sizes based on confidence intervals calculated for density estimates by DISTANCE. NR $=$ not recorded.

\begin{tabular}{|c|c|c|c|c|c|c|c|c|c|c|}
\hline Species & $\begin{array}{l}\text { Abra } \\
\text { Malaga } \\
0.13 \mathrm{~km}^{2}\end{array}$ & $\begin{array}{l}\text { Patacancha } \\
0.07 \mathrm{~km}^{2}\end{array}$ & $\begin{array}{l}\text { Huilloc } \\
0.09 \mathrm{~km}^{2}\end{array}$ & $\begin{array}{l}\text { Mantanay } \\
1.71 \mathrm{~km}^{2}\end{array}$ & $\begin{array}{l}\text { Pumahuanca } \\
0.39 \mathrm{~km}^{2}\end{array}$ & $\begin{array}{l}\text { San Juan } \\
0.13 \mathrm{~km}^{2}\end{array}$ & $\begin{array}{l}\text { Yanacocha } \\
0.13 \mathrm{~km}^{2}\end{array}$ & $\begin{array}{l}\text { Cancha- } \\
\text { Cancha } \\
0.27 \mathrm{~km}^{2}\end{array}$ & $\begin{array}{l}\text { Churo } \\
0.03 \mathrm{~km}^{2}\end{array}$ & $\begin{array}{l}\text { Maqui- } \\
\text { Maqui } \\
0.08 \mathrm{~km}^{2}\end{array}$ \\
\hline $\begin{array}{l}\text { Aglaeactis } \\
\text { castelnaudii }\end{array}$ & $6(5-9)$ & $3(2-5)$ & $4(3-6)$ & $82(57-116)$ & $19(13-27)$ & $6(4-9)$ & $6(5-9)$ & $13(9-18)$ & $1(1-2)$ & $4(3-5)$ \\
\hline $\begin{array}{l}\text { Oreotrochilus } \\
\text { estella }\end{array}$ & $1(1-3)$ & $I(0-2)$ & $I(1-2)$ & $18(9-40)$ & NR & NR & $1(1-3)$ & NR & $O(0-1)$ & $I(0-2)$ \\
\hline $\begin{array}{l}\text { Chalcostigma } \\
\text { stanleyi }\end{array}$ & $2(1-3)$ & NR & $I(1-2)$ & $21(10-43)$ & $5(2-10)$ & NR & $2(1-3)$ & $3(2-7)$ & $O(0-1)$ & $I(I-2)$ \\
\hline Colaptes rupicola & $1(0-11)$ & $1(0-6)$ & $1(0-8)$ & $14(1-144)$ & $3(0-33)$ & $1(0-11)$ & $1(0-11)$ & $2(2-3)$ & $\mathrm{O}(\mathrm{O}-2)$ & $1(0-7)$ \\
\hline Cinclodes aricomae & $0(0-0)$ & NR & $o(0-0)$ & $2(1-4)$ & $0(0-1)$ & NR & $\mathrm{o}(\mathrm{o}-\mathrm{o})$ & $0(0-1)$ & $o(0-0)$ & $\mathrm{o}(\mathrm{o}-\mathrm{o})$ \\
\hline Cinclodes fuscus & $I(0-2)$ & $0(0-1)$ & $1(0-1)$ & $11(5-24)$ & $3(1-6)$ & $I(0-2)$ & $1(0-2)$ & $2(1-4)$ & $o(o-o)$ & $1(0-1)$ \\
\hline $\begin{array}{l}\text { Leptasthenura } \\
\text { yanacensis }\end{array}$ & $2(1-3)$ & $1(1-1)$ & $I(1-2)$ & $20(12-36)$ & $5(3-8)$ & NR & $2(1-3)$ & $3(2-6)$ & $0(0-1)$ & $I(I-2)$ \\
\hline $\begin{array}{l}\text { Leptasthenura } \\
\text { xenothorax }\end{array}$ & $3(3-5)$ & $2(1-2)$ & $2(2-3)$ & $43(32-58)$ & $10(7-13)$ & NR & $3(3-5)$ & $7(5-9)$ & $1(1-1)$ & $2(2-3)$ \\
\hline $\begin{array}{l}\text { Cranioleuca } \\
\text { albicapilla }\end{array}$ & NR & NR & $I(1-2)$ & $24(14-41)$ & $5(3-9)$ & $2(1-3)$ & $2(1-3)$ & $4(2-7)$ & $O(O-1)$ & $I(I-2)$ \\
\hline Asthenes ottonis & $2(1-5)$ & $I(1-2)$ & $2(1-3)$ & $28(13-59)$ & $6(3-13)$ & $2(1-4)$ & $2(1-5)$ & $4(2-9)$ & $0(0-1)$ & $1(1-3)$ \\
\hline Asthenes humilis & $\mathrm{o}(\mathrm{o}-\mathrm{o})$ & $\mathrm{o}(\mathrm{o}-\mathrm{o})$ & $\mathrm{O}(\mathrm{o}-\mathrm{O})$ & $3(2-4)$ & NR & NR & $\mathrm{o}(\mathrm{o}-\mathrm{o})$ & NR & NR & NR \\
\hline $\begin{array}{l}\text { Asthenes } \\
\text { urubambensis }\end{array}$ & $0(0-1)$ & NR & $\mathrm{o}(\mathrm{o}-\mathrm{o})$ & $2(1-7)$ & $I(0-2)$ & $0(0-1)$ & $0(0-1)$ & $0(0-1)$ & $\mathrm{o}(\mathrm{o}-\mathrm{o})$ & $\mathrm{o}(\mathrm{o}-\mathrm{o})$ \\
\hline Asthenes virgata & $1(0-1)$ & $0(0-1)$ & $0(0-1)$ & $6(3-13)$ & NR & $0(0-1)$ & $1(0-1)$ & NR & NR & $0(0-1)$ \\
\hline Grallaria andicola & $0(0-1)$ & $o(o-o)$ & $o(0-0)$ & $3(2-8)$ & $1(0-2)$ & $1(0-1)$ & $0(0-1)$ & $1(0-1)$ & $o(o-o)$ & $o(0-o)$ \\
\hline Scytalopus simonsi & $I(0-2)$ & $1(0-1)$ & $1(0-1)$ & $10(6-19)$ & $2(1-5)$ & NR & $1(0-2)$ & $2(1-3)$ & $o(0-o)$ & $1(0-1)$ \\
\hline $\begin{array}{l}\text { Mecocerculus } \\
\text { leucophrys }\end{array}$ & NR & NR & $I(0-2)$ & $15(7-33)$ & $3(2-8)$ & NR & $1(1-3)$ & $2(1-5)$ & $0(0-1)$ & NR \\
\hline Anairetes alpinus & $1(0-1)$ & NR & $I(0-1)$ & $8(4-16)$ & $2(1-4)$ & NR & $1(0-1)$ & $I(1-3)$ & $\mathrm{o}(\mathrm{o}-\mathrm{o})$ & $0(0-1)$ \\
\hline Anairetes parulus & $2(1-3)$ & $1(1-2)$ & $I(I-2)$ & $25(16-41)$ & $6(4-9)$ & $2(1-3)$ & $2(1-3)$ & $4(3-6)$ & $0(0-1)$ & NR \\
\hline $\begin{array}{l}\text { Ochthoeca } \\
\text { rufipectoralis }\end{array}$ & $2(1-2)$ & $2(1-1)$ & $I(1-2)$ & $19(12-29)$ & $4(3-7)$ & $I(1-2)$ & $2(1-2)$ & $3(2-5)$ & $0(0-1)$ & $1(1-1)$ \\
\hline $\begin{array}{l}\text { Ochthoeca } \\
\text { oenanthoides }\end{array}$ & $\mathrm{o}(\mathrm{o}-\mathrm{o})$ & NR & $\mathrm{o}(\mathrm{o}-\mathrm{o})$ & $I(0-2)$ & $\mathrm{o}(\mathrm{o}-\mathrm{o})$ & $\mathrm{o}(\mathrm{o}-\mathrm{o})$ & $\mathrm{o}(\mathrm{o}-\mathrm{o})$ & $\mathrm{o}(\mathrm{o}-\mathrm{o})$ & $\mathrm{o}(\mathrm{o}-\mathrm{o})$ & NR \\
\hline
\end{tabular}


Table 4. Continued.

\begin{tabular}{|c|c|c|c|c|c|c|c|c|c|c|}
\hline Species & $\begin{array}{l}\text { Abra } \\
\text { Malaga } \\
0.13 \mathrm{~km}^{2}\end{array}$ & $\begin{array}{l}\text { Patacancha } \\
0.07 \mathrm{~km}^{2}\end{array}$ & $\begin{array}{l}\text { Huilloc } \\
0.09 \mathrm{~km}^{2}\end{array}$ & $\begin{array}{l}\text { Mantanay } \\
1.71 \mathrm{~km}^{2}\end{array}$ & $\begin{array}{l}\text { Pumahuanca } \\
0.39 \mathrm{~km}^{2}\end{array}$ & $\begin{array}{l}\text { San Juan } \\
0.13 \mathrm{~km}^{2}\end{array}$ & $\begin{array}{l}\text { Yanacocha } \\
0.13 \mathrm{~km}^{2}\end{array}$ & $\begin{array}{l}\text { Cancha- } \\
\text { Cancha } \\
0.27 \mathrm{~km}^{2}\end{array}$ & $\begin{array}{l}\text { Churo } \\
0.03 \mathrm{~km}^{2}\end{array}$ & $\begin{array}{l}\text { Maqui- } \\
\text { Maqui } \\
0.08 \mathrm{~km}^{2}\end{array}$ \\
\hline Troglodytes aedon & $I(1-1)$ & $0(0-1)$ & $I(0-1)$ & $9(6-13)$ & $2(1-3)$ & $1(1-1)$ & $1(1-1)$ & $I(1-2)$ & $\mathrm{o}(\mathrm{o}-\mathrm{o})$ & $0(0-1)$ \\
\hline Turdus chiguanco & $\mathrm{o}(\mathrm{o}-\mathrm{o})$ & $o(0-o)$ & $o(0-o)$ & $2(1-4)$ & $0(0-1)$ & $o(0-o)$ & $\mathrm{o}(\mathrm{o}-\mathrm{o})$ & $O(0-1)$ & $\mathrm{o}(\mathrm{o}-\mathrm{o})$ & $\mathrm{o}(\mathrm{o}-\mathrm{o})$ \\
\hline Turdus fuscater & NR & $0(0-1)$ & $0(0-1)$ & $6(3-12)$ & $1(1-3)$ & $1(0-1)$ & $I(0-1)$ & $1(1-2)$ & $o(o-o)$ & $O(0-1)$ \\
\hline $\begin{array}{l}\text { Conirostrum } \\
\text { cinereum }\end{array}$ & $4(2-7)$ & $2(1-4)$ & $3(1-5)$ & $48(25-93)$ & $11(6-21)$ & NR & $4(2-7)$ & $8(4-15)$ & $I(0-2)$ & $2(1-4)$ \\
\hline $\begin{array}{l}\text { Conirostrum } \\
\text { ferrugineiventre }\end{array}$ & $2(1-4)$ & $I(1-2)$ & $I(I-2)$ & $24(13-45)$ & $6(3-10)$ & NR & $2(I-4)$ & $4(2-7)$ & $0(0-1)$ & $I(1-2)$ \\
\hline Oreomanes fraseri & $3(2-4)$ & $2(1-2)$ & $2(2-3)$ & $38(28-51)$ & $9(6-12)$ & NR & $3(2-4)$ & $6(4-8)$ & $1(0-1)$ & $2(1-2)$ \\
\hline Xenodacnis parina & $3(2-5)$ & NR & $2(1-4)$ & $40(25-66)$ & $9(6-15)$ & $3(2-5)$ & $3(2-5)$ & $6(4-10)$ & $I(0-1)$ & $2(1-3)$ \\
\hline $\begin{array}{l}\text { Diglossa } \\
\text { brunneiventris }\end{array}$ & $0(0-1)$ & NR & $0(0-1)$ & $6(3-12)$ & $1(1-3)$ & NR & $0(0-1)$ & $I(0-2)$ & $\mathrm{o}(\mathrm{o}-\mathrm{o})$ & $O(0-1)$ \\
\hline Zonotrichia capensis & $S I(1-2)$ & $1(0-1)$ & $I(1-2)$ & $16(9-30)$ & $4(2-7)$ & $I(1-2)$ & $I(I-2)$ & $3(1-5)$ & $O(0-1)$ & $I(O-I)$ \\
\hline Phrygilus punensis & $1(1-1)$ & $0(0-1)$ & $1(0-1)$ & $10(7-15)$ & $2(2-3)$ & NR & $1(1-1)$ & $2(1-2)$ & $o(o-o)$ & $1(0-1)$ \\
\hline Phrygilus unicolor & $3(2-6)$ & $1(1-3)$ & $2(1-4)$ & $36(19-70)$ & $8(4-16)$ & $3(1-5)$ & $3(2-6)$ & $6(3-11)$ & $I(0-1)$ & $2(1-3)$ \\
\hline Phrygilus plebejus & $1(0-3)$ & $1(0-2)$ & $I(0-2)$ & $15(5-42)$ & $3(1-10)$ & $1(0-3)$ & $1(0-3)$ & $2(1-7)$ & $0(0-1)$ & $I(0-2)$ \\
\hline Idiopsar brachyurus & NR & NR & $O(0-2)$ & $8(2-33)$ & NR & NR & $1(0-3)$ & $1(1-5)$ & NR & NR \\
\hline Catamenia inornata & $I(0-1)$ & $\mathrm{o}(\mathrm{o}-\mathrm{o})$ & $0(0-1)$ & $7(5-11)$ & $2(1-3)$ & $1(0-1)$ & $1(0-1)$ & $I(I-2)$ & $\mathrm{o}(\mathrm{o}-\mathrm{o})$ & $O(0-1)$ \\
\hline Carduelis atrata & $1(1-2)$ & $1(0-1)$ & $1(1-1)$ & $15(9-25)$ & $3(2-6)$ & $1(1-2)$ & $1(1-2)$ & $2(1-4)$ & $o(o-o)$ & $1(0-1)$ \\
\hline $\begin{array}{l}\text { Carduelis } \\
\text { crassirostris }\end{array}$ & $I(I-2)$ & $1(0-1)$ & $I(1-1)$ & $17(11-27)$ & $4(2-6)$ & NR & $I(1-2)$ & $3(2-4)$ & $\mathrm{o}(\mathrm{o}-\mathrm{o})$ & $I(1-2)$ \\
\hline
\end{tabular}


forest cover (e.g. Churo). All population estimates were extremely low, even in the areas with the greatest forest cover. No species had a population estimate greater than 82 individuals $(A$. castelnaudii at Mantanay). Of the six globally-threatened species, population estimates did not exceed 43 individuals (L. xenothorax at Mantanay).

\section{Discussion}

My results confirm what has become a general pattern for forest-dependent bird communities in fragmented landscapes: higher species richness and diversity in larger forest patches, and low densities of individual species. Densities of Polylepis bird species were greater in larger forest patches and differed significantly between different patch size categories. Similar declines in bird density with decreasing forest patch size following habitat fragmentation have been recorded in a number of studies (e.g. Willis 1979, Laurance 1991, Newmark 1991, Radford and Bennett 2004) with low density species being at greater risk (Stouffer and Bierregaard 1995, Stratford and Stouffer 1999). Applying the same criteria to the Polylepis bird communities of the Cordillera Vilcanota suggests that the species most at risk from future habitat loss are $C$. aricomae, $A$. urubambensis, A. alpinus, G. andicola and S. simonsi (Table 3).

Density estimates for the majority of matrix-dependent species were higher in smaller Polylepis patches (Table 3). This is an important finding, indicating that the matrix exerts a significant influence on bird species composition and abundance in Polylepis forests, particularly smaller patches. Matrix habitat is often the most extensive and connected element in fragmented forest landscapes (Forman 1997) and forest patches must be examined as part of a wider, dynamic landscape (Lindenmayer et al. 2002, Wethered and Lawes 2003). Matrix habitat management will therefore be an integral component of optimizing Polylepis conservation efforts in the Cordillera Vilcanota.

I was unable to calculate density estimates for both A. urubambensis and $A$. virgata in any of the three forest patch size categories due to insufficient sample sizes. A. urubambensis has often been considered as being 'common' at a number of Polylepis forest sites, particularly at the northern end of the Cordillera Vilcanota where the species shows a distinctive preference for bushy P. pepei or P. sericea forests on cloud-enshrouded ridges (Walker and Fjeldså 2005, Fjeldså pers. comm). Some authors have commented that populations of A. urubambensis are almost certainly declining due to ongoing loss of Polylepis and elfin forest habitats (e.g. Stotz et al. 1996, Birdlife International 2004, Remsen 2003) but the species can be often overlooked due to its skulking behaviour (Fjeldså pers. comm). The paucity of records for A. urubambensis during the survey probably reflects the genuine rarity of the species at the three study sites. This rarity and that similar numbers of the forest-matrix interface species $A$. virgata were also recorded in all forest patch size categories merit investigation.

There are limitations to this study, mainly how representative are these results of other high Andean regions, and accounting for potential sources of bias in the census methodology. Individual bird species have specific habitat requirements and I acknowledge that there may be considerable variation in densities and population estimates of individual species related to habitat quality and climate (e.g. humidity) in different high Andean areas. Consequently, densities and population estimates of some of the globally threatened species may be higher in other Polylepis areas.

One potential sources of bias in the census methodology is whether all individuals for all species were detected efficiently, particularly skulking species (e.g. S. simonsi) that may have moved from the census plots during the five minute settling period. Failure to record these species would violate one of the main assumptions of Distance Sampling (see Buckland et al. 1993). Another potential source of bias concerns whether the higher density estimates for the majority of matrix-dependent species in smaller Polylepis patches than in either large or medium patches, is simply an artefact of habitat quality i.e. is it possible that the methodology performed better in smaller forest patches because they are more open, with less dense vegetation cover, due to greater 
logging activity. Individual birds foraging across small forest patches are generally more mobile and this could result in higher encounter rates in small patches rather than in large or medium patches. Further analysis of settling down periods, length of counts, and influence of habitat quality on encounter rates are required to explore these possibilities.

\section{Patterns of rarity and implications for conservation}

The density estimates for Polylepis birds are fairly low, and are lower still in small forest patches, but are Polylepis bird species intrinsically low density species? There are no quantitative population surveys of other Andean habitat specialists with which to compare the Polylepis density estimates. Further insight may therefore be provided by comparing these densities with those of lowland habitat specialists. Kratter (1995) used spot-mapping to estimate densities of insectivorous birds restricted to patches of Guadua bamboo in three different lowland forest habitats. In order to create a classification compatible with Kratter's spot-mapping study, I have adapted three categories of rarity based on rarity criteria designated by Terborgh et al. (1990): ( 1 ) resident bird species with measured density estimates of $\leqslant 2$ individuals but $\geqslant 1$ individual $\mathrm{km}^{-2} ;(2)$ resident species with densities too low to estimate $\left(\leqslant 0.5\right.$ individual $\left.\mathrm{km}^{-2}\right) ;(3)$ overall rarity (category $1+$ category 2 ).

A number of conclusions can be made from the analysis in Table 5. In general, patterns of rarity varied within each habitat specialist bird community, and between Polylepis and Guadua bamboo habitats. Within the Guadua bamboo community, the largest number of rare species in both category 1 and 2, were found in river-edge bamboo, than in bluff-top or floodplain bamboo habitat. Overall, the majority of bamboo specialists were not intrinsically rare, with $74-95 \%$ of species abundant (high density estimate per unit area of habitat) within this rare and patchy lowland habitat (Kratter 1995).

In the first rarity category, a greater number and percentage of Polylepis birds are considered rare in large and medium patches, and in all patch sizes combined, compared with both small Polylepis patches, and the three different Guadua bamboo habitats. However, this is only a relatively small number and percentage of the Polylepis bird community. The most noticeable patterns of rarity within the Polylepis community are evident in both the second and third categories. A greater number of Polylepis birds are considered category 2 rarities in small forest patches, than bamboo birds in all three lowland bamboo habitats. The category 3 results show

Table 5. correlates of rarity in two Neotropical habitat specialist bird communities. Data are number of species. Figures in parenthesis represent percentage of species' totals. Data on bamboo birds * from Kratter (1995) and converted to individual $\mathrm{km}^{-2}$. Rarity categories adapted from Terborgh et al. (1990). Birds classified as dependent, to a degree, on Polylepis forests $\uparrow$ from (Lloyd 2007).

\begin{tabular}{|c|c|c|c|}
\hline & $\begin{array}{l}\text { CATEGORY } \mathbf{1} \\
\text { Density } \leqslant 2 \text { individuals } \\
\text { but } \geqslant 1 \text { individual } \mathrm{km}^{-2} \text {. }\end{array}$ & $\begin{array}{l}\text { CATEGORY } 2 \text { Densities } \\
\text { too low to estimate } \\
\left(\leqslant 0.5 \text { individual } \mathrm{km}^{-2}\right)\end{array}$ & OVERALL RARITY \\
\hline \multicolumn{4}{|l|}{$\begin{array}{l}\text { Bamboo birds (19 } \\
\text { species) * }\end{array}$} \\
\hline Bluff-top bamboo & $1(5 \%)$ & o & $1(5 \%)$ \\
\hline Floodplain bamboo & o & $2(11 \%)$ & $2(11 \%)$ \\
\hline River-edge bamboo & o & $5(26 \%)$ & $5(26 \%)$ \\
\hline \multicolumn{4}{|l|}{$\begin{array}{l}\text { Polylepis birds ( } 27 \\
\text { species) } \dagger\end{array}$} \\
\hline Combined estimate & $5(19 \%)$ & o & $5(19 \%)$ \\
\hline Large patch estimate & $3(11 \%)$ & $3(11 \%)$ & $6(22 \%)$ \\
\hline Medium patch estimate & $5(19 \%)$ & $4(15 \%)$ & $9(34 \%)$ \\
\hline Small patch estimate & $I(4 \%)$ & $19(70 \%)$ & $20(74 \%)$ \\
\hline
\end{tabular}


that, overall, Polylepis birds are rarer than lowland Guadua bamboo birds. More importantly, it shows that between $19-22 \%$ of species are intrinsically rare species within their rare and highly fragmented habitat, and that a greater number of Polylepis bird species (between $34-74 \%$ of all species) become rarer in smaller forest patches. Declines in the densities of certain Polylepis birds may therefore be predictable following habitat loss and these patterns of rarity should govern population recovery goals through appropriate habitat restoration strategies.

\section{Habitat restoration in the Cordillera Vilcanota}

Populations of all Polylepis birds are extremely low in the remaining forest areas of the Cordillera Vilcanota, and are probably much lower than had been suspected by previous authors (e.g. Fjeldså 1988, 1993, Fjeldså and Kessler 1996). For some bird species, this is certainly not a cause for concern. Many species, e.g. C. rupicola, A. ottonis, A. parulus, C. cinereum, T. fuscater, T. chiguanco, P. punensis, and C. atrata, are found in a variety of widespread, and less threatened Andean habitats (see Fjeldså and Kessler 1996 and Parker et al. 1996). What is of great concern, are the extremely low population estimates for all six globally-threatened species (Table 4). This analysis of abundance and rarity shows that there is no substitute for the quantity of habitat needed to support viable populations of threatened, low-density, Polylepis bird species throughout the Cordillera Vilcanota. Habitat restoration strategies designed to prevent further forest habitat loss and increase forest habitat quantity in areas where Polylepis forest previously existed are urgently required to boost populations of these threatened species. The Peruvian organisation Asociación Ecosistemas Andinos (ECOAN) is currently pioneering such an approach, and as a result of their efforts, Polylepis habitat restoration efforts are currently underway at a small number of locations in the Cordillera Vilcanota.

\section{Acknowledgements}

I would like to thank Barry and Rosario Walker of Manu Expeditions, and the Department of Environmental and Geographical Sciences, Manchester Metropolitan University, for sponsoring the research. For their help and support in Perú, I would like to thank Constantino Aucca and the staff of ECOAN, Eliana Manga, Freddy Padovani, Navidad Abandanio, Mary Montesinos, and the Department of Immigration (Cusco Region - Perú). Luc Lens, Jon Fjeldså and Paul Salaman greatly improved earlier versions of the manuscript.

\section{Bibliography}

Ambuel, B. and Temple, S. A. (1983) Areadependent changes in the bird communities and vegetation of southern Wisconsin forests. Ecology 64: 1057-1068.

Antongiovanni, M. and Metzger, J. P. (2005) Influence of matrix habitats on the occurrence of insectivorous bird species in Amazonian forest fragments. Biol. Conserv. 122: 441-451.

Aucca, C., Ferro Meza, G., Samochuallpa, E. and Palomino, W. (2001) Conservation action plan for Polylepis relict forests of southern Peru. Unpublished ECOAN Report.
Bibby, C. J., Burgess, N. D., Hill, D. A. and Mustoe, S. H. (2000) Bird census techniques (second edition). London: Academic Press.

BirdLife International (2004) Threatened birds of the world 2004 CD-ROM. Cambridge, U.K.: BirdLife International.

Blake, J. G. and Karr, J. R. (1984) Species composition of bird communities and the conservation benefit of large versus small forests. Biol. Conserv. 30: 173-187.

Blake, J. G. and Karr, J. R. (1987) Breeding birds of isolated woodlots: area and habitat relationships. Ecology 68: 1724-1734. 
Borgella, R. and T. A. Gavin. (2005) Avian community dynamics in a fragmented tropical landscape. Ecol. Applic. 15: 1062-1073.

Buckland, S. T., Anderson, D. R., Burnham, K. P. and Laake, J. L. (1993) Distance sampling: Estimating abundance of biological populations. New York: Chapman and Hall.

Buckland, S. T., Anderson, D. R., Burnham, K. P., Laake, J. L., Borchers, D. L. and Thomas, L. (2001) Introduction to distance sampling: Estimating abundance of biological populations. Oxford, U.K.: Oxford University Press.

Colwell, R. K. (2005) EstimateS: Statistical estimation of species richness and shared species from samples. Version $7.5<\mathrm{http}: / /$ viceroy.eeb.uconn.edu/EstimateS $>$

Colwell, R. K. and Coddington, J. A. (1994) Estimating terrestrial biodiversity through extrapolation. Phil. Trans. R. Soc. B. 345: 101-118.

Diamond, J. M. (1975) Assembly of species communities. Pp. 342-444 in M. L. Cody and J. M. Diamond, eds. Ecology and evolution of communities. Cambridge, Massachusetts: Harvard University Press.

Driscoll, D. A. (2005) Is the matrix a sea? Habitat specificity in a naturally fragmented landscape. Ecol. Entomol. 30: 8-16.

Fischer, J., Fazey, I., Briese, R. and D. B. Lindenmayer, D. B. (2005) Making the matrix matter: challenges in Australian grazing landscapes. Biodiv. and Conserv. 14: 561-578.

Fjeldså, J. (1988) Status of birds in steppe habitats of the Andean Zone and Patagonia. Pp. 81-95 in P. D. Goriup, ed. Ecology and conservation of grassland birds. Cambridge, U.K.: International Council for Bird Preservation. Technical Publication No. 7 .

Fjeldså, J. (1993) The avifauna of the Polylepis woodlands of the Andean highlands: the efficiency of basing conservation priorities on patterns of endemism. Bird Conserv. Internat. 3: 37-55.

Fjeldså, J. (2002) Polylepis forests - vestiges of a vanishing ecosystem in the Andes. Ecotropica 8: 111-123.
Fjeldså, J. and Kessler, M. (1996) A strategy for conserving the biological diversity of Polylepis woodlands of the High Andes in Peru and Bolivia. Copenhagen: CTB/ NORDECO.

Forman, R. T. T. (1997) Land mosaics: The ecology of landscapes and regions. Cambridge, UK: Cambridge University Press.

Gascon, C., Lovejoy, T. E., Bierregaard, R. O., Malcolm, J. R., Stouffer, P. C., Vasconcelos, H., Laurance, W. F., Zimmerman, B., Tocher, M. and Borges, S. (1999) Matrix habitat and species persistence in tropical forest remnants. Biol. Conserv. 91: 223-230.

Jacobs, M. D. and Walker, J. S. (1999) Density estimates of birds inhabiting fragments of cloud forest in southern Ecuador. Bird Conserv. Internat. 9: 73-80.

James, F. C. and Rathburn, S. (1981) Rarefaction, relative abundances, and diversity of avian communities. The Auk 98: 785-8oo.

Jones, M. J., Lindsey, M. D. and Marsden, S. J. (1995) Population sizes, status and habitat associations of the restricted-range bird species of Sumba, Indonesia. Bird Conserv. Internat. 5: 21-52.

Kessler, M. (2002) The "Polylepis problem": where do we stand? Ecotropica 8: 97-110.

Kratter, A. W. (1995) Bamboo specialization in Amazonian birds. Ph.D dissertation. Baton Rouge, Louisiana: Louisiana State University.

Lægaard, S. (1992) Influence of fire in the grass páramo vegetation of Ecuador. Pp. $151-170$ in H. Balslev and J. L. Luteyn, eds. Páramo: an Andean ecosystem under human influence. London: Academic Press.

Laurance, W. F. (1991) Edge effects in tropical forest fragments: application of a model for the design of nature reserves. Biol. Conserv. 57: 205-219.

Laurance, W. F. (1994) Rainforest fragmentation and the structure of small mammal communities in tropical Queensland. Biol. Conserv. 69: 23-32.

Lee, D. C. (2005) Improving methods for conservation-based assessments of abundance and habitat use in tropical birds. 
Ph.D dissertation. Manchester, UK: Manchester Metropolitan University.

Lindenmayer, D. B., Cunningham, R. B., Donnelly, C. F., Nix, H. and Lindenmayer, B. D. (2002) Effects of forest fragmentation on bird assemblages in a novel landscape context. Ecol. Monogr. 72: I-18.

Lloyd, H. (200o) Population densities of the Black-faced Cotinga Conioptilon mcilhen$n y i$ in southeast Peru. Bird Conserv. Internat. 10: 277-285.

Lloyd, H. (2004) Habitat and population estimates of some threatened lowland forest bird species in Tambopata, southeast Peru. Bird Conserv. Internat. 14: 261-277.

Lloyd, H. (2007) Abundance and ecology of high-Andean Polylepis birds in Perú: implications for habitat management strategies. Ph.D. dissertation. Manchester, UK: Manchester Metropolitan University.

Lloyd, H. and Marsden, S. J. (in press) Bird community variation across Polylepis woodland fragments and matrix habitats: implications for conservation within a high Andean landscape. Biodiv. and Conserv.

Magurran, A. E. (2004) Measuring biological diversity. Oxford, UK: Blackwell Publishing.

Marsden, S. J. (1999) Estimation of parrot and hornbill densities using a point count distance sampling method. Ibis 141: 377-390.

Marsden, S. J., Whiffin, M. and Galetti, M. (2001) Bird diversity and abundance in forest fragments and Eucalyptus plantations around an Atlantic forest reserve, Brazil. Biodiv. and Conserv. 10: 737-751.

Marsden, S. J., Whiffin, M., Galetti, M. and Fielding, A. H. (2005) How well will Brazil's system of Atlantic Forest reserves maintain viable bird populations? Biodiv. and Conserv. 14: 2835-2853.

Newmark, W. D. (1991) Tropical forest fragmentation and the local extinction of understorey birds in the Eastern Usambara Mountains, Tanzania. Conserv. Biol. 5: $67-78$.

Parker, T. A., Stotz, D. F., Fitzpatrick, J. W. (1996) Ecological and distributional databases for Neotropical birds. Pp. 113-436 in
D. F. Stotz, J. W. Fitzpatrick, T. A. Parker and D. K. Moskovits. Neotropical birds: ecology and conservation. Chicago: The University of Chicago Press.

Peters, R. H. (1991) A critique for ecology. Cambridge, UK: Cambridge University Press.

Radford, J. Q. and Bennett, A. F. (2004) Thresholds in landscape parameters: occurrence of the white-browed treecreeper Climacteris affinis in Victoria, Australia. Biol. Conserv. 117: 375-391.

Remsen, J. V. (2003) Family Furnariidae (ovenbirds). Pp. 162-357 in J. del Hoyo, A. Elliott and D. A. Christie, eds. Handbook of the birds of the world. Vol. 8. Broadbills to tapaculos. Barcelona: Lynx Edicions.

Renison, D., Hensen, I. and Cingolani, A. M. (2004) Anthropogenic soil degradation affects seed viability in Polylepis australis mountain forests of central Argentina. Forest Ecol. Manage. 196: 327-333.

Reynolds, R. T., Scott, J. M. and Nussbaum, R. A. (1980) A variable circular-plot method for estimating bird numbers. Condor 82: 309-313.

Rivera-Milan, F. F., Ruiz, C. A., Cruz, J. A. and Sustache, J. A. (2003) Reproduction of plain pigeons (Columba inornata wetmorei) in east-central Puerto Rico. The Auk 120: 466-480.

Simberloff, D. (1972) Properties of the rarefaction diversity measurement. Am. Nat. 106: 414-418.

Smallwood, K. S. (2001) Linking habitat restoration to meaningful units of animal demography. Restoration Ecol. 9: 253-261.

Smith, E. P. and van Belle, G. (1984) Nonparametric estimation of species richness. Biometrics 40: 119-129.

Stotz, D. F., Fitzpatrick, J. W., Parker, T. A. and Moskovits, D. K. (1996) Neotropical birds: ecology and conservation. Chicago: The University of Chicago Press.

Stouffer, P. C. and Bierregaard, B. O. (1995) Use of Amazonian forest fragments by understorey insectivorous birds. Ecology 76: 2429-2445.

Stratford, J. A. and Stouffer, P. C. (1999) Local extinction of terrestrial insectivorous birds in a fragmented landscape near Manaus, Brazil. Conserv. Biol. 13: 1416-1423. 
Tear, T. H., Scott, J. M., Hayward, P. H. and Griffith, B. (1995) Recovery plans and the Endangered Species Act: are criticisms supported by data? Conserv. Biol. 9: 182-195.

Teich, I., Cingolani, A. M., Renison, D., Hensen, I. and Giorgis, M. A. (2005) Do domestic herbivores retard Polylepis australis Bitt. woodland recovery in the mountains of Cordoba, Argentina? Forest Ecol. Manage. 219: 229-241.

Terborgh, J. W., Robinson, S. K., Parker, T. A., Munn, C. A. and Pierpont, N. (1990) The structure and organization of an Amazonian forest bird community. Ecol. Monogr. 60: 213-238.

Thomas, L., Laake, J. L., Strindberg, S., Marques, F. F. C., Buckland, S. T.,
Borchers, D. L., Anderson, D. R., Burnham, K. P., Hedley, S. L., Pollard, J. H. and Bishop, J. R. B. (2004) Distance 4.1. St. Andrews, UK: Research Unit for Wildlife Population Assessment, University of St. Andrews.

Walker, B. and Fjeldså, J. (2005) . Field Guide to the birds of Machu Picchu and the Cusco regionLima: Nuevas Imagines S.A.

Wethered, R. and Lawes, M. J. (2003) Nestedness of bird assemblages in fragmented Afromontane forest: the effect of plantation forestry in the matrix. Biol. Conserv. 123: 125-137.

Willis, E. O. (1979) The composition of avian communities in remanescent woodlots in southern Brazil. Papéis Avulsos de Zoologia 33: 1-25.

\section{HUW LLOYD}

Department of Environmental and Geographical Sciences, Manchester Metropolitan University, Chester Street, Manchester, MI 5GD, U.K. e-mail: h.lloyd@mmu.ac.uk

Received 21 March 2007; revision accepted 31 August 2007 